\title{
Sistema no invasivo para la medida y visualización de desplazamientos de tejidos en neurocirugía
}

\author{
Juan, Carlos G.; Blanco-Angulo, Carolina; Bermejo, Natividad; García, Hector; \\ Vicente, Jose María; Avila, Ernesto; Sabater-Navarro, Jose María \\ Grupo de Neuroingeniería Biomédica de la Universidad Miguel Hernández de Elche.
}

\begin{abstract}
A medical imaging system based on measuring distances between tissues by means of microwaves is presented. The system is designed for the monitoring and correction of the brain-shift problem in brain tumor operations. It shows the developed system and the first results with phantoms of brain tissues that have a morphology similar to real tissues. The viability of the system for the measurement of distances and the reconstruction of an intraoperative $3 D$ image is demonstrated.
\end{abstract}

Keywords: bioengineering, brain-shift, technology, microwave.

\section{Resumen}

Se presenta un sistema de imagen médica basado en medición de distancias entre tejidos por medio de microondas. El sistema está pensado para la monitorización y corrección del problema de brain-shift en operaciones de tumores cerebrales. Se muestra el sistema desarrollado y los primeros resultados con phantoms de tejidos cerebrales que tienen una morfología similar a los tejidos reales. Se demuestra la viabilidad del sistema para la medida de distancias y la reconstrucción de una imagen $3 D$ intraoperatoria.

Palabras clave: bioingeniería, brain-shift, tecnología, microondas. 


\section{Introducción}

La neurocirugía robótica está sufriendo profundos cambios en los últimos tiempos, fruto principalmente de los avances en las técnicas de imagen médica (TAC, MRI, f-MRI o DTI), lo que permite una mejor planificación de la operación a realizar. La neurocirugía mínimamente invasiva se ve beneficiada de estos avances. Sin embargo, quedan problemas a resolver en la transferencia del plan de trabajo preplanificado a la realidad intraoperatoria, debido a la naturaleza no lineal de los tejidos deformables involucrados. Uno de estos problemas es el brain-shift, o desplazamiento de la materia cerebral fruto del cambio de presión interior al practicar la craneotomía y de los propios procesos quirúrgicos, y que produce una pérdida de referencia entre los volúmenes de imagen de neurocirugía adquiridos antes de la cirugía.

Diversos autores han propuesto soluciones para abordar este problema. En (Letteboer, 2005) se propone el uso de un sistema de ecografía 3D que permita obtener una imagen 3D del volumen del cerebro. Otras aproximaciones han hecho uso de sistemas ópticos (Comparetti, 2011) para trackear los puntos anatómicos, si bien esta aproximación tiene la limitación de que el marker debe ser visible. Más interesantes son las aproximaciones que hacen uso de un modelo matemático de deformaciones (DeLorenzo, 2012, Chen, 2013, Oldfield, 2011) para predecir los desplazamientos de todos los puntos del cerebro.

En este trabajo se presenta un sistema de imagen médica por microondas para detección y corrección del problema de brain-shift en operaciones de tumores cerebrales. Se presenta el sistema desarrollado, los componentes electrónicos de alta frecuencia y control que forman parte del mismo y el software que permite obtener las imágenes. El sistema de antenas permite obtener en tiempo real la localización de los puntos de control del modelo de deformaciones para la visualización utilizando realidad aumentada.

\section{Metodología}

El sistema propuesto para realizar las medidas de distancias entre la antena y los diferentes tejidos biológicos creaneales se basa en un sistema de antenas de radiofrecuencia (RF) que permiten crear una imagen médica mediante la aplicación de algortimos de reconstrucción de imagen médica. La Figura 1 muestra una imagen del set-up prototipo donde se observan los principales componentes. 


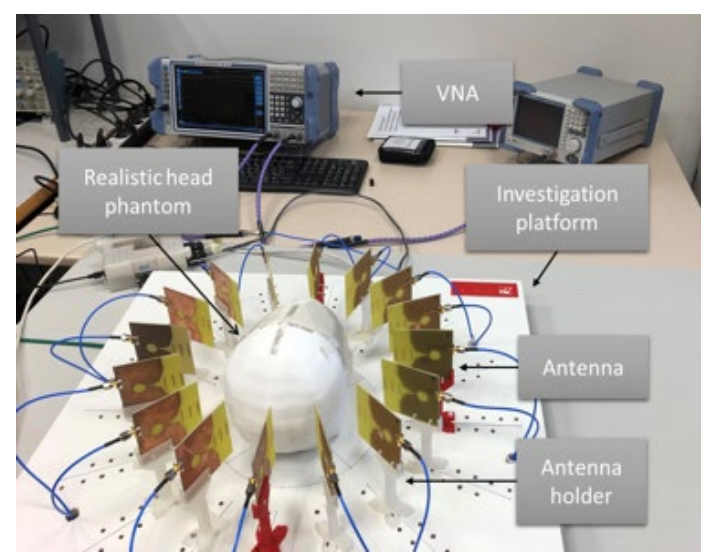

Fig. 1 Fotografía del sistema utilizado para obtener la imagen médica donde se pueden observar los distintos elementos que componen el sistema. Fuente: Elaboración propia (2019).

\subsection{Antenas diseñadas}

Las antenas, que se han diseñado y fabricado para el sistema de medida del proyecto son antenas tipo Vivaldi impresas en sustrato FR4 de $7 \mathrm{~cm}^{2}$. En la Figura 2 se muestran ambas caras de la antena diseñada. Como se puede observar, son antenas de tipo slot a la que se han añadido 3 elementos directores en su apertura y un radial stub $\lambda / 4$ en la alimentación. Estos elementos se han optimizado durante el proceso de diseño para obtener la mejor relación entre tamaño y diversos parámetros de la antena como ancho de banda, directividad y ganancia. En concreto los tres elementos directores situados en la apertura de la antena aumentan la directividad y ganancia, y se han diseñado para optimizar el diagrama de radiación a la frecuencia central de funcionamiento. Para alimentar la antena, como se puede ver en la imagen, se ha diseñado una línea microstrip seguida del radial stub $\lambda / 4$ que proporciona la adaptación de banda ancha. En este trabajo se utilizan 16 antenas colocadas formando un círculo y separadas $22.5^{\circ}$ entre sí respecto al centro de la circunferencia.
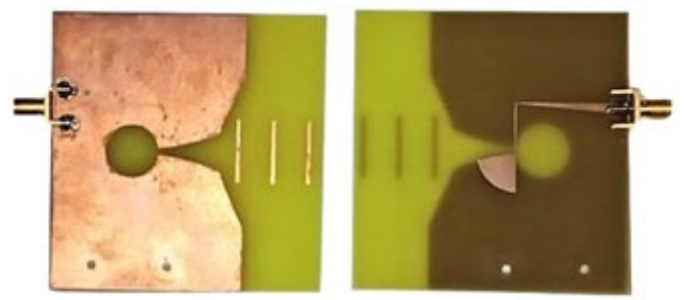

Fig. 2 Fotografía del prototipo de antena fabricado. Fuente: Elaboración propia (2018).

La antena se ha diseñado y simulado con el software EMPro. En la Figura 3 se muestra el parámetro S11 en el rango de funcionamiento, tanto de la simulación en EMPro como en la 
medida de la antena fabricada. Como se puede observar, la antena presenta un ancho de banda de funcionamiento de $1.2 \mathrm{GHz}$ a $5 \mathrm{GHz}$, medido para una respuesta por debajo de $-10 \mathrm{~dB}$.

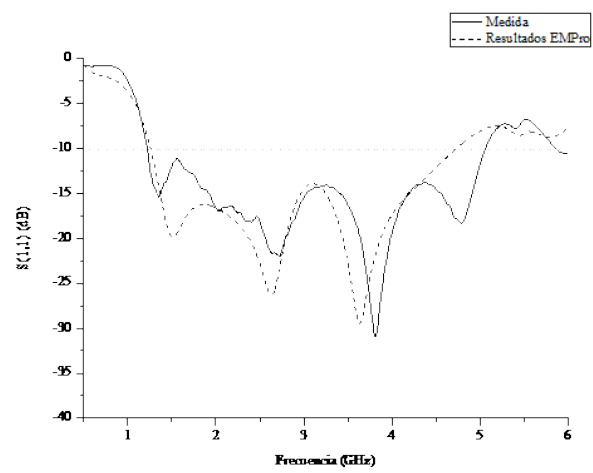

Fig. 3 Pérdidas de retorno de la antena fabricada. Fuente: Elaboración propia (2018).

En la Figura 4 se muestran los diagramas de radiación de la antena en plano E y plano $\mathrm{H}$, respectivamente. Estos diagramas se han representado para cinco frecuencias dentro del ancho de banda de funcionamiento de la antena (1, 2, 3, 4 y $5 \mathrm{GHz})$. Se puede extraer que la antena es prácticamente omnidireccional en el plano E en todo el rango. Por otro lado, en el plano $\mathrm{H}$ la antena es más directiva en la dirección de la apertura, gracias a los elementos directores colocados en esa dirección.

Por último, cabe comentar que la ganancia simulada con el software EMPro oscila entre 3 y $7 \mathrm{~dB}$ en todo el rango de frecuencias de funcionamiento de la antena.

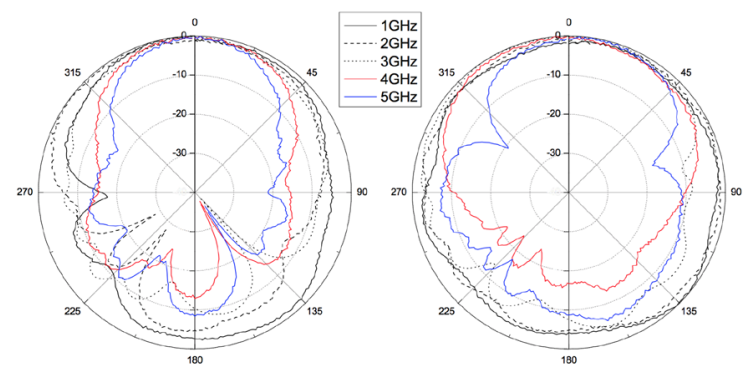

Fig. 4 Diagramas de radiación medidos de la antena en plano H (a) y E (b). Fuente: Elaboración propia (2018).

\subsection{Sistema de medida de distancias}

Debajo del tablero de madera que se observa en la Figura 1 se encuentra una red de 5 conmutadores de RF que seleccionan qué antena de las 16 utilizadas está conectada al VNA 
y mide en cada momento. Para las medidas se utiliza un phantom como el descrito en (Bermejo, 2018), donde se especifican las recetas para obtener phantoms de tejidos con propiedades dieléctricas en el rango de los tejidos biológicos, de forma que se pueda comprobar el funcionamiento del sistema desarrollado. La Tabla 1 muestra los parámetros de los tejidos phantoms utilizados.

Tabla 1. Parámetros dieléctricos de los phantoms utilizados.

\begin{tabular}{|c|c|c|}
\hline Materiales & $\boldsymbol{\varepsilon}^{\prime}$ & $\operatorname{tg} \boldsymbol{\delta}$ \\
\hline Piel & 44 & 0,64 \\
\hline Cerebro & 50 & 0,54 \\
\hline Tumor & 75 & 0,18 \\
\hline
\end{tabular}

\subsubsection{Commutadores de RF}

La conmutación de las antenas se realiza mediante conmutadores de RF. En concreto, se utilizan 5 conmutadores modelo ZSWA4-63DR+ de Mini-Circuits. Este tipo de conmutador se compone de una entrada y 4 salidas de RF que operan en la banda de $1 \mathrm{MHz}$ a $6 \mathrm{GHz}$ y están adaptadas a $50 \Omega$ en toda la banda.

El fabricante proporciona una tabla de verdad en la que relaciona la entrada con las salidas. En la tabla también se indican dos estados adicionales, uno de ellos es el estado de reposo de los conmutadores y el otro es un estado no soportado, el cual no debería producirse.

De estos cinco conmutadores, uno de ellos es el conmutador que controla los otros cuatro, formando una topología de estrella, siendo además el conmutador que se conecta al VNA para medir los resultados. Cada uno de los otros cuatro conmutadores está conectado a cuatro antenas, dividiendo así la circunferencia de 16 antenas en cuatro secciones gobernadas por un conmutador cada una. Las señales de control y alimentación provienen de la placa de control y se conectan mediante cables tipo DB9.

\subsubsection{Sistema de control}

Para el control de los conmutadores y alimentación de los mismos, el sistema dispone de un microprocesador basado en la plataforma Arduino Due y una placa de control diseñada para facilitar las conexiones de los conectores DB9.

El microcontrolador recibe las instrucciones programadas en Python y se alimenta a través del puerto USB del ordenador. Las salidas digitales de Arduino, conectadas a la placa de control que ha sido diseñada y fabricada para este trabajo, proporcionan la codificación 
necesaria en cada caso a los conmutadores mediante los cables DB9. Para la alimentación de los conmutadores se ha añadido un pequeño regulador de tensión, de forma que toda la alimentación necesaria en el sistema la proporciona el puerto USB. Por último, la placa incluye unos LEDs que muestran en todo momento la codificación que están recibiendo cada uno de los conmutadores.

\subsection{Algoritmo de visualización de la deformación del tejido}

En la Figura 5 se presenta el esquema del algoritmo computacional utilizado para reconstruir la imagen 3D del cerebro, localizar con precisión las anomalías cerebrales en la cabeza mediante la adquisición de imágenes MRI y visualizar utilizando realidad aumentada (RA) las deformaciones geométricas de los órganos encefálicos en tiempo real. Una vez definidos los modelos anatómicos geométricos, se expone la ubicación de los puntos de control en la superficie cerebral que proporcionarán información en tiempo real de su ubicación. También se expone el método de conexión de dichos puntos con el modelo cerebral completo. Además, se muestra el resultado de los hologramas 3D para visualizar en un sistema de RA unos desplazamientos hipotéticos ocurridos a los tejidos biológicos cerebrales.

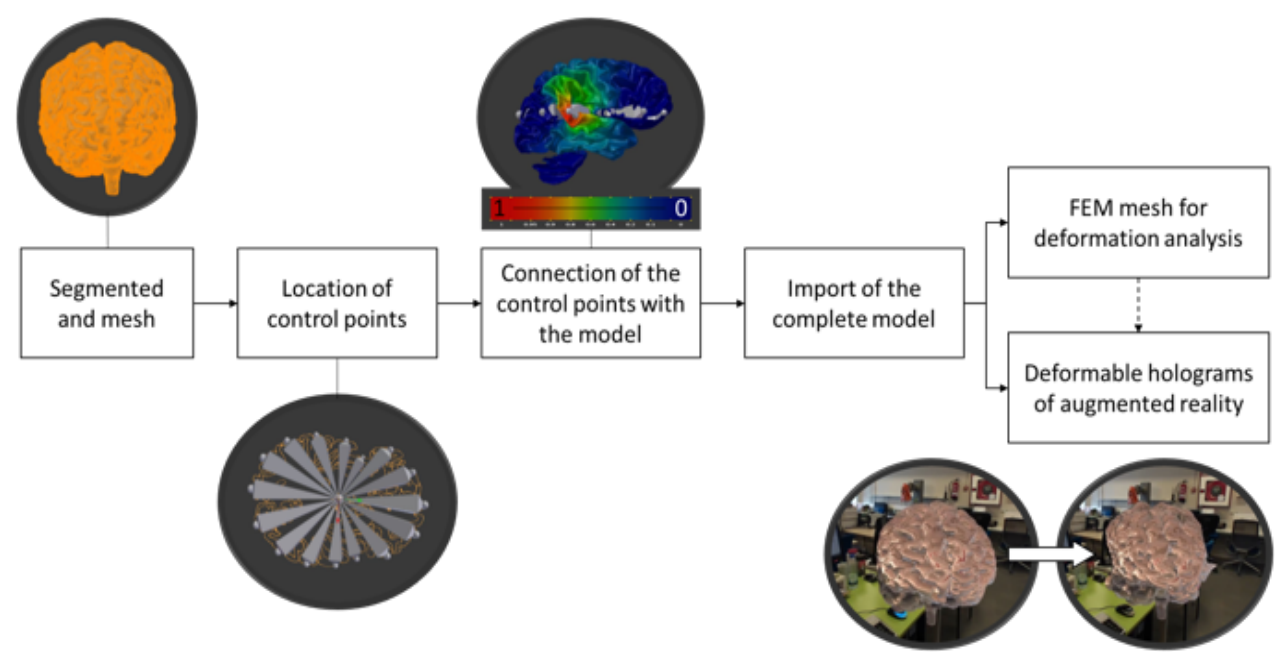

Fig. 5 Esquema para visualización 3D del brain-shift. Fuente: Elaboración propia (2018).

\subsubsection{Segmentación y mallado}

Un paso importante para distinguir órganos, tumores, o cualquier detalle anatómico es la segmentación. Se trata de una de las operaciones más usadas en el procesado digital de imágenes en 3D. Consiste en asignar a cada píxel una clasificación sobre el tipo de tejido que 
representa. Con esta información se pueden definir las superficies de los órganos o lesiones para poder hacer una reconstrucción 3D.

En este trabajo, la segmentación ha sido realizada utilizando un atlas cerebral multimodal basado en imágenes MRI disponible en la página web del Surgical Planning Laboratory (Halle, 2019).

\subsubsection{Ubicación de los puntos de información en la superficie cerebral}

En un trabajo anterior (Bermejo, 2018), se evaluó en un set-up con una geometría sencilla el sistema de medida de distancias usando señales de radiofrecuencia mediante un sistema experimental preliminar. Consistía en las mediciones de una antena para obtener las medidas en el dominio de la frecuencia de distancias entre planos. En el presente trabajo, se presenta una evolución que consiste en utilizar una geometría más compleja, basada en 3D, y similar a la disposición geométrica de los tejidos cerebrales. Se realiza el seguimiento de 16 puntos de control sobre la superficie del tejido phantom.

\section{Resultados}

Se han realizado diversas pruebas del sistema diseñado, utilizando el modelo de cerebro indicado en el apartado 1.2 e introduciendo dentro de la mezcla y en diferentes ubicaciones, un pequeño recipiente de plástico diseñado con impresora 3D que contiene un volumen de $0.5 \mathrm{ml}$ de agua marina. En la Figura 6 se puede ver la solución de la imagen en coordenadas cartesianas de una de las pruebas. En este caso, el elemento extraño se colocó aproximadamente a $200^{\circ}$. Analizando la imagen se puede extraer que el objeto está ubicado a $200^{\circ}$ ya las antenas más cercanas a esta posición reciben un rebote antes que las demás (alrededor de unos $2 \mathrm{~ns}$ ) y con una mayor intensidad. El resto de antenas prácticamente no ven el elemento ya que el material utilizado es muy absorbente.

Para mejorar la calidad de la imagen generada se ha utilizado el algoritmo de Delay-And-Sum (DAS). Esta técnica nos permite detectar variaciones anormales en el dieléctrico a partir de la información de amplitud y fase de la señal recibida. La imagen final se genera a partir del desfase de las señales y la posterior suma de cada uno de los puntos de la imagen a generar. En el algoritmo DAS, la región de interés se divide en una pequeña rejilla o puntos focales. Para cada uno de esos puntos, se calcula la distancia entre los puntos y la antena. A partir de ese valor obtenemos los retardos de las señales y se genera la imagen. En la Figura 7 se representa la imagen de un objeto extraño colocado en $270^{\circ}$ aplicando este algoritmo. 


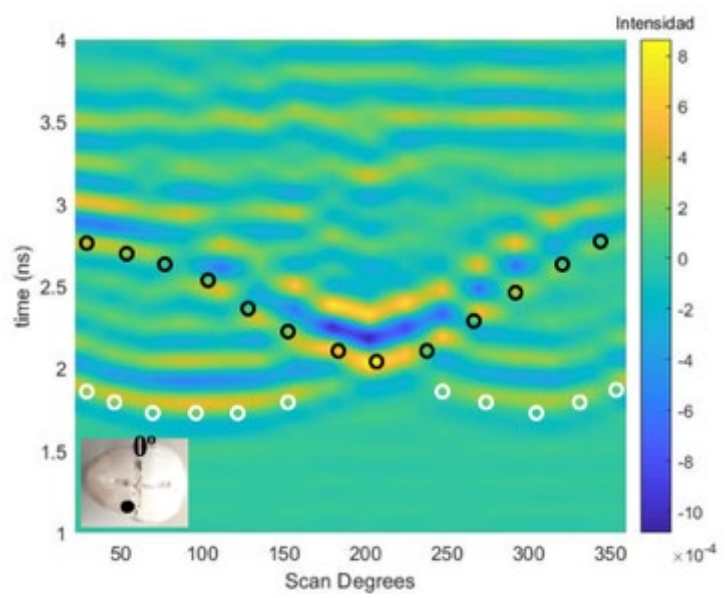

Fig. 6 Resultados de una sesión experimental..Fuente: Elaboración propia (2019).

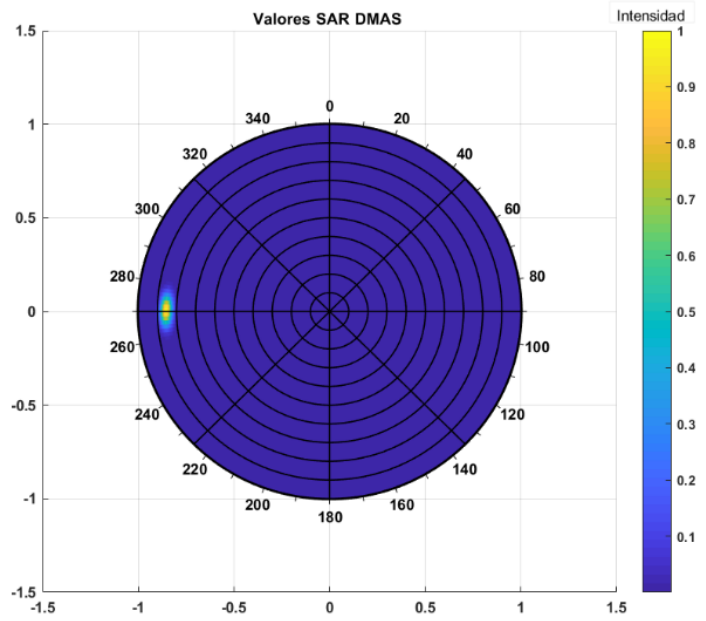

Fig. 7 Resultado en coordenadas circulares con artefacto en 270..Fuente: Elaboración propia (2019).

\section{Conclusiones}

En este trabajo se ha presentado un sistema basado en antenas de banda ancha a frecuencias de microondas para la medida de distancias en deformaciones del tejido cerebral. El sistema está formado por 16 antenas Vivaldi de reducidas dimensiones que trabajan en el rango de 1-5 GHz. Para la conexión de cada antena al analizador vectorial de redes se ha diseñado un 
sistema de conmutación de alta frecuencia controlado por un microcontrolador que, a su vez, está gestionado por un ordenador, donde se procesa la información de cada una de las antenas y se genera la imagen. Para probar el sistema desarrollado se ha utilizado un modelo de cerebro fabricado con materiales de uso general y que presenta propiedades dieléctricas similares al tejido cerebral.

El dispositivo se ha testado en varios supuestos prácticos, utilizando como modelo de tumor agua marina, presentando en todos los casos un buen funcionamiento. El dispositivo desarrollado es capaz de detectar y ubicar artefactos de pequeñas dimensiones, por debajo de $0.5 \mathrm{ml}$ de volumen.

\section{Referencias}

BERMEJO et al. (2018), "Description of the experimental model for the measurement of distance in biological tissues from RF signals," en Spanish Robotics Conference, Valladolid, España.

CHEN, R. E. et al. (2012), "Integrating Retraction Modeling Into an Atlas-Based Framework for Brain Shift Prediction," en IEEE Transactions on Biomedical Engineering, vol. 60, no. 12, pp. 3494-3504.

COMPARETTI M. D. et al. (2011), "Optically tracked multi-robot system for keyhole neurosurgery," en 2011 IEEE International Conference on Robotics and Automation (ICRA), Shanghai, China, pp. 661-666.

DELORENZO, X. et al. (2012), "Volumetric Intraoperative Brain Deformation Compensation: Model Development and Phantom Validation," en IEEE Transactions on Medical Imaging, vol. 31, no. 8, pp. 1607-1619.

HALLE M. et al. (2019) "Multi-modality MRI-based Atlas of the Brain," en SPL 2017 Jan. [en línea]. Disponible en: http://www.spl.harvard.edu/publications/item/view/2037. [Fecha acceso: 12 de febrero de 2019].

LETTEBOER, M. M. J. et al. (2005), "Brain shift estimation in image-guided neurosurgery using 3-D ultrasound," en IEEE Transactions on Biomedical Engineering, vol. 52, no. 2, pp. 268-276.

OLDFIELD, M. J. et al, (2011), "Detailed finite element modelling of deep needle insertions into a soft tissue phantom using a cohesive approach," en Computer Methods in Biomechanics and Biomedical Engineering, vol. 16, no. 5, pp. 530-543. 